\title{
PI-PROPERTIES OF SOME MATRIX ALGEBRAS WITH INVOLUTION
}

\author{
TSETSKA RASHKOVA
}

Received 23 September, 2015

\begin{abstract}
We define the nilpotency index of the b-variables in second order matrix algebras with Grassmann entries and involution $b$. Identities of minimal degree are found for a concrete subalgebra of the matrix algebra $M_{4}(K)$. When it has an involution $\phi$ as well some of its $\phi$-identities are given. For an analogue of this subalgebra over finite dimensional Grassmann algebras a new involution (b) is introduced and its (b)-identities are discussed.
\end{abstract}

2010 Mathematics Subject Classification: 16R10; 15A75; 16R50

Keywords: PI-algebras, matrix algebra over Grassmann algebras, algebras with involution $\phi$, $\phi$-variables, $\phi$-identities

\section{INTRODUCTION}

The classical PI-theory (the theory of the polynomial identities) has its development for algebras with involution as well. The contributions of Amitsur [1], Levchenko [9], Rowen [14], Wenxin and Racine [17], Giambruno and Valenti [6], Drensky and Giambruno [5], Rashkova [11], La Mattina and Misso [8] are only a part of it.

In 1973 Krasovski and Regev [7] described completely the $T$-ideal of the identities of the Grassmann algebra $E$ and it was a natural step to investigate the PI-structure of algebras not only over fields (with any characteristic) but over algebras as well, especially Grassmann algebras $[4,12,16]$.

In the paper we consider mainly finite dimensional Grassmann algebras and special matrix algebras over them.

We recall the definition of the Grassmann algebra $E$ as:

$$
E=K\left\langle e_{1}, e_{2}, \ldots \mid e_{i} e_{j}+e_{j} e_{i}=0, i, j=1,2, \ldots\right\rangle,
$$

where $K$ is a field of characteristic zero.

We cite basic propositions from $[3,7]$. The notation $[x, y, z]=[[x, y], z]=[x, y] z-$ $z[x, y]$ will be used.

Partially supported by Grant I 02/18 of the Bulgarian National Science Fund. 
Proposition 1 ([7, Corollary, p. 437]). The T - ideal of the Grassmann algebra E is generated by the identity $[x, y, z]=0$.

Proposition 2 ([3, Lemma 6.1]). For any $n, k \geq 2$ in the algebra $E$ the identity $S_{n}^{k}\left(x_{1}, \ldots, x_{n}\right)=0$ holds, where

$$
S_{n}\left(x_{1}, \ldots, x_{n}\right)=\sum_{\sigma \in \operatorname{Sym}(n)}(-1)^{\sigma} x_{\sigma(1)} \ldots x_{\sigma(n)}
$$

is the $n$-th standard polynomial.

Proposition 3 ([3, Lemma 6.6]). The matrix algebra $M_{n}(E)$ does not satisfy the identity

$$
S_{m}^{n}\left(x_{1}, \ldots, x_{m}\right)=0
$$

for any $m$.

There are subalgebras of $M_{n}(E)$ however being counter examples of Proposition 3 for concrete $m$.

We use the notation $E_{n}^{\prime}$ for a non unitary Grassmann algebra with generators $e_{1}, \ldots, e_{n}$.

The existence of nilpotent elements of minimal nilpotency index both in finite dimensional Grassmann algebras and in matrix algebras over them was investigated in $[12,13]$. We state some of the results needed:

Proposition 4 ([13, Proposition 13]). The identity $x^{3}=0$ holds for the algebra $E_{4}^{\prime}$.

Proposition 5 ([13, Proposition 16]). The algebra $M_{2}\left(E_{4}^{\prime}\right)$ satisfies the identity $X^{4}=0$.

In [13] examples were given as well of subalgebras $\mathfrak{A}_{i}, i=1,2$ of $M_{n}(\mathfrak{R})$ such that the identities $x^{4}=0$ and $[x, y, z]=0$ in $\mathfrak{R}$ imply the identity $X^{4}=0$ in $\mathfrak{A}_{\mathfrak{i}}, i=1,2$.

An involution $\psi$ on the Grassmann algebras $E_{2}^{\prime}$ and $E_{3}^{\prime}$ defines an involution $\phi$ on the corresponding $2 \times 2$ matrix algebra over any of them. In that case the classes of symmetric and of skew-symmetric to the involution $\phi$ matrices of nilpotency indices 2 and 3 were described in [12].

In the present paper we continue the investigations started in [12]:

We define the nilpotency index of the $b$-variables in the considered algebras with involution $\phi=b$.

For a concrete subalgebra of the matrix algebra $M_{4}(K)$ identities of minimal degree are found. When additionally the algebra has an involution $\phi$ some of its $\phi$ identities are given.

For an analogue of this subalgebra over finite dimensional Grassmann algebras a new involution $\phi=(b)$ is introduced and some (b)-identities are discussed. 


\section{Results}

\subsection{PI-properties of involution second order matrix algebras with Grassmann} entries

We recall the definition of an involution on an algebra $R$ : it is a second order antiautomorphism $\psi$ such that $\psi(a b)=\psi(b) \psi(a)$ for all $a, b \in R$.

By $R^{-}$we denote the skew-symmetric due to the involution elements of $R$, namely $z_{1}, \ldots, z_{i}, \ldots$ and by $R^{+}$we denote the symmetric due to the involution elements $y_{1}, \ldots, y_{j}, \ldots$. It is important to consider $\psi$-variables (symmetric and skew-symmetric) as the elements of $R^{+}$form a Jordan algebra due to the multiplication $y_{1} \circ y_{2}=$ $y_{1} y_{2}+y_{2} y_{1}$ and the elements of $R^{-}$form a Lie algebra due to the operation $\left[z_{1}, z_{2}\right]$.

Definition 1. Let $f=f\left(x_{1}, \ldots, x_{m}\right) \in K\left\langle x_{1}, \ldots, x_{n}\right\rangle$, the free associative algebra on $n$ generators over $K$. We say that $f$ is a $\psi$-identity in skew variables for the algebra $R$ over $K$ if $f\left(z_{1}, \ldots, z_{m}\right)=0$ for all $z_{1}, \ldots, z_{m} \in R^{-}$. Accordingly $f$ is a $\psi$-identity in symmetric variables for the algebra $R$ over $K$ if $f\left(y_{1}, \ldots, y_{m}\right)=0$ for all $y_{1}, \ldots, y_{m} \in R^{+}$.

We say that $f$ is a $\psi$-identity if $f\left(z_{1}, \ldots, z_{i}, y_{i+1}, \ldots, y_{m}\right)=0$ for any $z_{1}, \ldots, z_{i} \in$ $R^{-}$and any $y_{i+1}, \ldots, y_{m} \in R^{+}$.

We denote an involution on the basic field or algebra as $\psi$ while $\phi$ will mean an involution on the corresponding matrix algebra.

If a ring $R$ has an involution $\psi=*$ two involutions $\phi_{1}=\sharp$ and $\phi_{2}=b$ on $M_{2}(R)$ are defined as follows [15]:

$$
\left(\begin{array}{ll}
a & b \\
c & d
\end{array}\right)^{\#}=\left(\begin{array}{ll}
a^{*} & c^{*} \\
b^{*} & d^{*}
\end{array}\right),\left(\begin{array}{ll}
a & b \\
c & d
\end{array}\right)^{b}=\left(\begin{array}{ll}
d^{*} & b^{*} \\
c^{*} & a^{*}
\end{array}\right) .
$$

It is known [2] that two involutions play an important role in the Grassmann algebra: the involution $\psi_{1}$ acting on the generators $e_{i}$ of $E$ as $\psi_{1}\left(e_{2 k}\right)=e_{2 k-1}$, $\psi_{1}\left(e_{2 k-1}\right)=e_{2 k}$ and the trivial on the generators involution $\psi_{2}$ for which $\psi_{2}\left(e_{i}\right)=$ $e_{i}$ for all $e_{i}$

Here we consider the algebra $\left(M_{2}\left(E_{4}^{\prime}, \psi_{2}\right), b\right)$ and continue some of the investigations made in [12] by finding the nilpotency index of the b-variables of $\left(M_{2}\left(E_{4}^{\prime}, \psi_{2}\right), b\right)$.

Theorem 1. The algebra $\left(M_{2}\left(E_{4}^{\prime}, \psi_{2}\right), b\right)$ satisfies the b-identity $Y^{4}=0$ in bsymmetric variables and the b-identity $Z^{3}=0$ in b-skew symmetric variables.

Proof of Theorem 1. As Proposition 5 holds we have to prove only that $Z^{3}=0$ in b-skew symmetric variables.

Let $Z=\left(\begin{array}{ll}y_{1} & z_{1} \\ z_{2} & y_{2}\end{array}\right)$. The condition $\phi_{2}(Z)=-Z$ means that $\psi_{2}\left(z_{1}\right)=-z_{1}$, $\psi_{2}\left(z_{2}\right)=-z_{2}, \psi_{2}\left(y_{1}\right)=-y_{2}$ and $\psi_{2}\left(y_{2}\right)=-y_{1}$. Thus we get that

$$
z_{1}=\alpha_{5} e_{1} e_{2}+\alpha_{6} e_{1} e_{3}+\alpha_{7} e_{1} e_{4}+\alpha_{8} e_{2} e_{3}+\alpha_{9} e_{2} e_{4}+\alpha_{10} e_{3} e_{4}
$$




$$
\begin{aligned}
& +\alpha_{11} e_{1} e_{2} e_{3}+\alpha_{12} e_{1} e_{2} e_{4}+\alpha_{13} e_{1} e_{3} e_{4}+\alpha_{14} e_{2} e_{3} e_{4} ; \\
z_{2} & =\beta_{5} e_{1} e_{2}+\beta_{6} e_{1} e_{3}+\beta_{7} e_{1} e_{4}+\beta_{8} e_{2} e_{3}+\beta_{9} e_{2} e_{4}+\beta_{10} e_{3} e_{4} \\
& +\beta_{11} e_{1} e_{2} e_{3}+\beta_{12} e_{1} e_{2} e_{4}+\beta_{13} e_{1} e_{3} e_{4}+\beta_{14} e_{2} e_{3} e_{4} ; \\
y_{1} & =\gamma_{1} e_{1}+\gamma_{2} e_{2}+\gamma_{3} e_{3}+\gamma_{4} e_{4} \\
& +\gamma_{5} e_{1} e_{2}+\gamma_{6} e_{1} e_{3}+\gamma_{7} e_{1} e_{4}+\gamma_{8} e_{2} e_{3}+\gamma_{9} e_{2} e_{4}+\gamma_{10} e_{3} e_{4} \\
& +\gamma_{11} e_{1} e_{2} e_{3}+\gamma_{12} e_{1} e_{2} e_{4}+\gamma_{13} e_{1} e_{3} e_{4}+\gamma_{14} e_{2} e_{3} e_{4}+\gamma_{15} e_{1} e_{2} e_{3} e_{4} ; \\
y_{2} & =-\gamma_{1} e_{1}-\gamma_{2} e_{2}-\gamma_{3} e_{3}-\gamma_{4} e_{4} \\
& +\gamma_{5} e_{1} e_{2}+\gamma_{6} e_{1} e_{3}+\gamma_{7} e_{1} e_{4}+\gamma_{8} e_{2} e_{3}+\gamma_{9} e_{2} e_{4}+\gamma_{10} e_{3} e_{4} \\
& +\gamma_{11} e_{1} e_{2} e_{3}+\gamma_{12} e_{1} e_{2} e_{4}+\gamma_{13} e_{1} e_{3} e_{4}+\gamma_{14} e_{2} e_{3} e_{4}-\gamma_{15} e_{1} e_{2} e_{3} e_{4} .
\end{aligned}
$$

As in $z_{i} z_{j}$ the least degree of the summands is 4 we have $x z_{j} z_{k}=0, z_{j} x z_{k}=0$, $z_{j} z_{k} x=0$ for any entry $x$ of the matrix $Z$. As the least degree of the summands in $y_{i} z_{j}$ is 3 we get that $y_{i} z_{j} z_{k}=0$. The least degree in $y_{i}^{2}$ is 3 and we have $y_{i}^{2} z_{j}=0$ and $z_{i} y_{j}^{2}=0$ as well. Thus for the matrix $Z^{3}=\left(a_{i j}\right)$ we get $a_{11}=a_{22}=0, a_{12}=$ $y_{1} z_{1} y_{2}$ and $a_{21}=y_{2} z_{2} y_{1}$.

We consider the four summands of degree 3 (the minimal one) in $y_{1} z_{1}$ :

$$
\begin{aligned}
& \alpha e_{1} e_{2} e_{3} \rightarrow \alpha=\gamma_{1} \alpha_{8}-\gamma_{2} \alpha_{6}+\gamma_{3} \alpha_{5} \\
& \beta e_{1} e_{2} e_{3} \rightarrow \beta=\gamma_{1} \alpha_{9}-\gamma_{2} \alpha_{7}+\gamma_{4} \alpha_{5} \\
& \gamma e_{1} e_{2} e_{3} \rightarrow \gamma=\gamma_{1} \alpha_{10}-\gamma_{3} \alpha_{7}+\gamma_{4} \alpha_{6} \\
& \delta e_{1} e_{2} e_{3} \rightarrow \delta=\gamma_{2} \alpha_{10}-\gamma_{3} \alpha_{9}+\gamma_{4} \alpha_{8} .
\end{aligned}
$$

Now we define the coefficient of the only summand (of degree 4) in $a_{12}=y_{1} z_{1} y_{2}$. It is equal to

$$
\begin{aligned}
& -\gamma_{4}\left(\gamma_{1} \alpha_{8}-\gamma_{2} \alpha_{6}+\gamma_{3} \alpha_{5}\right)+\gamma_{3}\left(\gamma_{1} \alpha_{9}-\gamma_{2} \alpha_{7}+\gamma_{4} \alpha_{5}\right) \\
& -\gamma_{2}\left(\gamma_{1} \alpha_{10}-\gamma_{3} \alpha_{7}+\gamma_{4} \alpha_{6}\right)+\gamma_{1}\left(\gamma_{2} \alpha_{10}-\gamma_{3} \alpha_{9}+\gamma_{4} \alpha_{8}\right) \equiv 0 .
\end{aligned}
$$

The same is valid for $a_{21}=y_{2} z_{2} y_{1}$ as well. Thus $Z^{3}$ is the zero matrix.

If we change the involution $\psi_{2}$, considered in $E_{4}^{\prime}$, with the involution $\psi_{1}$, the b-variables of $\left(M_{2}\left(E_{4}^{\prime}, \psi_{1}\right), b\right)$ do not have a lower nilpotency index, namely

Theorem 2. The algebra $\left(M_{2}\left(E_{4}^{\prime}, \psi_{1}\right), b\right)$ satisfies the b-identity $A^{4}=0$ for $A$ being any b-variable.

Proof of Theorem 2. We mach only the crucial steps of the proof.

In this case $\psi_{1}\left(e_{1}\right)=e_{2}\left(\psi_{1}\left(e_{2}\right)=e_{1}\right)$ and $\psi_{1}\left(e_{3}\right)=e_{4}\left(\psi_{1}\left(e_{4}\right)=e_{3}\right)$.

We have to consider only the case when $A=Z$ is a $b$-skew symmetric variable. The conditions $\psi_{1}\left(z_{i}\right)=-z_{i}$ and $\psi_{1}\left(y_{1}\right)=-y_{2}$ give that

$$
\begin{aligned}
z_{1} & =\alpha_{1}\left(e_{1}-e_{2}\right)+\alpha_{3}\left(e_{3}-e_{4}\right)+\alpha_{6}\left(e_{1} e_{3}+e_{2} e_{4}\right)+\alpha_{7}\left(e_{1} e_{4}+e_{2} e_{3}\right) \\
& +\alpha_{11}\left(e_{1} e_{2} e_{3}-e_{1} e_{2} e_{4}\right)+\alpha_{13}\left(e_{1} e_{3} e_{4}-e_{2} e_{3} e_{4}\right) \\
z_{2} & =\beta_{1}\left(e_{1}-e_{2}\right)+\beta_{3}\left(e_{3}-e_{4}\right)+\beta_{6}\left(e_{1} e_{3}+e_{2} e_{4}\right)+\beta_{7}\left(e_{1} e_{4}+e_{2} e_{3}\right)
\end{aligned}
$$




$$
\begin{aligned}
& +\beta_{11}\left(e_{1} e_{2} e_{3}-e_{1} e_{2} e_{4}\right)+\beta_{13}\left(e_{1} e_{3} e_{4}-e_{2} e_{3} e_{4}\right) ; \\
y_{1} & =\gamma_{1} e_{1}+\gamma_{2} e_{2}+\gamma_{3} e_{3}+\gamma_{4} e_{4} \\
& +\gamma_{5} e_{1} e_{2}+\gamma_{6} e_{1} e_{3}+\gamma_{7} e_{1} e_{4}+\gamma_{8} e_{2} e_{3}+\gamma_{9} e_{2} e_{4}+\gamma_{10} e_{3} e_{4} \\
& +\gamma_{11} e_{1} e_{2} e_{3}+\gamma_{12} e_{1} e_{2} e_{4}+\gamma_{13} e_{1} e_{3} e_{4}+\gamma_{14} e_{2} e_{3} e_{4} ; \\
y_{2} & =-\gamma_{2} e_{1}-\gamma_{1} e_{2}-\gamma_{4} e_{3}-\gamma_{3} e_{4} \\
& -\gamma_{5} e_{1} e_{2}+\gamma_{9} e_{1} e_{3}+\gamma_{8} e_{1} e_{4}+\gamma_{7} e_{2} e_{3}+\gamma_{6} e_{2} e_{4}-\gamma_{10} e_{3} e_{4} \\
& -\gamma_{12} e_{1} e_{2} e_{3}-\gamma_{11} e_{1} e_{2} e_{4}-\gamma_{14} e_{1} e_{3} e_{4}-\gamma_{13} e_{2} e_{3} e_{4} .
\end{aligned}
$$

We follow the coefficient of $e_{1} e_{2} e_{3}$ in the entry $a_{11}=z_{1} z_{2} y_{1}+y_{1} z_{1} z_{2}+z_{1} y_{2} z_{2}$ of the matrix $Z^{3}=\left(a_{i j}\right)$. Forming $z_{1} z_{2}$ we find the coefficient of $e_{1} e_{2} e_{3}$ in the product $y_{1}\left(z_{1} z_{2}\right)$, namely $-\left(\gamma_{1}+\gamma_{2}\right)\left(\alpha_{1} \beta_{3}-\alpha_{3} \beta_{1}\right)$.

The same holds for the coefficient of $e_{1} e_{2} e_{3}$ in the products $z_{1} z_{2} y_{1}$ and in $z_{1} y_{2} z_{2}$. Thus $Z^{3}$ is not a zero matrix.

Taking into account the conditions on the entries of a b-symmetric matrix $Y$ we see that the coefficient of $e_{1} e_{2} e_{3}$ in the entry $b_{11}$ of the matrix $Y^{3}=\left(b_{i j}\right)$ is $3\left(\gamma_{1}-\right.$ $\left.\gamma_{2}\right)\left(\alpha_{1} \beta_{3}-\alpha_{3} \beta_{1}\right)$.

\subsection{PI-properties of some fourth order matrix algebras}

We define the 8-th dimensional matrix algebra $A M_{4}(K)$ as the algebra of the matrices of type

$$
\left(\begin{array}{cccc}
a_{11} & 0 & a_{13} & 0 \\
0 & a_{22} & 0 & a_{24} \\
a_{31} & 0 & a_{33} & 0 \\
0 & a_{42} & 0 & a_{44}
\end{array}\right), a_{i j} \in K \text {. The following theorem holds: }
$$

Theorem 3. The algebra $A M_{4}(K)$ satisfies the Hall identity $\left[\left[X_{1}, X_{2}\right]^{2}, X_{3}\right]=0$.

Proof of Theorem 3. For $X_{1}, X_{2} \in A M_{4}(K)$ in $\left[X_{1}, X_{2}\right]=\left(c_{i j}\right)$ we have $c_{33}=$ $-c_{11}$ and $c_{44}=-c_{22}$. The matrix $\left[X_{1}, X_{2}\right]^{2}=\left(d_{i j}\right)$ is a diagonal matrix with $d_{33}=$ $d_{11}$ and $d_{44}=d_{22}$. Thus $\left[\left[X_{1}, X_{2}\right]^{2}, X_{3}\right]=0$.

By the system for computer algebra Mathematica we see that $A M_{4}(K)$ satisfies the identity $S_{4}\left(X_{1}, X_{2}, X_{3}, X_{4}\right)=0$ as well.

The n-th analogue of $A M_{4}(K)$ is the algebra $A M_{2 n}(K)$. Its elements are of type $\left(a_{i j}\right)$ with non-zero entries only among $a_{i i}$ for $i=1, \ldots, 2 n, a_{j, n+j}$ and $a_{n+j, j}$ for $j=1, \ldots, n$. The two identities in $A M_{4}(K)$ hold in $A M_{2 n}(K)$ as well.

It is known that in a matrix algebra over a field $K$ of characteristic zero up to isomorphism there are two types of involutions - the transpose one $t$ and the symplectic involution $*$, the latter defined on an even $2 k$ order matrix algebra as

$$
\left(\begin{array}{cc}
A & B \\
C & D
\end{array}\right)^{*}=\left(\begin{array}{cc}
D & -B^{t} \\
-C^{t} & A
\end{array}\right),
$$


where $A, B, C, D$ are $k \times k$ matrices.

We recall that the Hall identity $\left[\left[Y_{1}, Y_{2}\right]^{2}, Y_{3}\right]=0$ is a $*$-identity of minimal degree in $*$-symmetric variables for the algebra $\left(M_{4}(K), *\right)$ [5].

Next we consider the matrix algebra $A M_{4}(K)$ with the symplectic involution $*$.

Theorem 4. The algebra $\left(A M_{4}(K), *\right)$ satisfies the $*$-identity $\left[Y_{1}, Y_{2}\right]=0$ in $*$ symmetric variables.

Proof of Theorem 4. From

$$
\begin{aligned}
& \left(\begin{array}{cccc}
a_{11} & 0 & a_{13} & 0 \\
0 & a_{22} & 0 & a_{24} \\
a_{31} & 0 & a_{33} & 0 \\
0 & a_{42} & 0 & a_{44}
\end{array}\right){ }^{*} \\
& =\left(\begin{array}{cccc}
a_{33} & 0 & -a_{13} & 0 \\
0 & a_{44} & 0 & -a_{24} \\
-a_{31} & 0 & a_{11} & 0 \\
0 & -a_{42} & 0 & a_{22}
\end{array}\right)=\left(\begin{array}{cccc}
a_{11} & 0 & a_{13} & 0 \\
0 & a_{22} & 0 & a_{24} \\
a_{31} & 0 & a_{33} & 0 \\
0 & a_{42} & 0 & a_{44}
\end{array}\right)
\end{aligned}
$$

we see that the $*$-symmetric elements of $\left(A M_{4}(K), *\right)$ are diagonal matrices.

As $z^{2}$ is $*$-symmetric we come to

Corollary 1. The algebra $\left(A M_{4}(K), *\right)$ satisfies the $*$-identity $\left[Z_{1}^{2}, Z_{2}^{2}\right]=0$ in *-skew symmetric variables.

Now the matrix algebras considered will have entries that are elements of a Grassmann algebra. In the statements below we use Proposition 4. As it was proved in [13] using the system for computer algebra Mathematica we give here its analytic proof.

Proof of Proposition 4. Without loss of generality we consider $x \in E_{4}^{\prime}$ with summands of length 1 and 2 only (the other ones will give zeros either in $x^{2}$ or in $x^{3}$ ). Thus

$$
\begin{aligned}
x & =\alpha_{1} e_{1}+\alpha_{2} e_{2}+\alpha_{3} e_{3}+\alpha_{4} e_{4}+\alpha_{5} e_{1} e_{2}+\alpha_{6} e_{1} e_{3} \\
& +\alpha_{7} e_{1} e_{4}+\alpha_{8} e_{2} e_{3}+\alpha_{9} e_{2} e_{4}+\alpha_{10} e_{3} e_{4} .
\end{aligned}
$$

We define the coefficients of the four summands of length 3 in $x^{2}$. They are:

$$
\begin{aligned}
& \alpha e_{1} e_{2} e_{3} \mapsto \alpha=2\left(\alpha_{1} \alpha_{8}-\alpha_{2} \alpha_{6}+\alpha_{3} \alpha_{5}\right) \\
& \beta e_{1} e_{2} e_{4} \mapsto \beta=2\left(\alpha_{1} \alpha_{9}-\alpha_{2} \alpha_{7}+\alpha_{4} \alpha_{5}\right) \\
& \gamma e_{1} e_{3} e_{4} \mapsto \gamma=2\left(\alpha_{1} \alpha_{10}-\alpha_{3} \alpha_{7}+\alpha_{4} \alpha_{6}\right) \\
& \delta e_{2} e_{3} e_{4} \mapsto \delta=2\left(\alpha_{2} \alpha_{10}-\alpha_{3} \alpha_{9}+\alpha_{4} \alpha_{8}\right) .
\end{aligned}
$$

The coefficient of the only summand (which is of length 4 ) of $x^{3}$ is proportional to

$$
\begin{aligned}
& -\alpha_{1}\left(\alpha_{2} \alpha_{10}-\alpha_{3} \alpha_{9}+\alpha_{4} \alpha_{8}\right)+\alpha_{2}\left(\alpha_{1} \alpha_{10}-\alpha_{3} \alpha_{7}+\alpha_{4} \alpha_{6}\right) \\
& -\alpha_{3}\left(\alpha_{1} \alpha_{9}-\alpha_{2} \alpha_{7}+\alpha_{4} \alpha_{5}\right)+\alpha_{4}\left(\alpha_{1} \alpha_{8}-\alpha_{2} \alpha_{6}+\alpha_{3} \alpha_{5}\right) \equiv 0
\end{aligned}
$$


The identity $[y, x, x]=0$ and the linearization of $x^{3}=0$ lead to

Corollary 2. In $E_{4}^{\prime}$ the following identities hold:

$$
\begin{aligned}
& x^{2} y+y x^{2}=0, x y x=0, x y z+z y x=0, \\
& x y^{2} z=-z y x y=0, y^{2} x z=-z y x y=0, z x y^{2}=-y x y z=0 .
\end{aligned}
$$

Theorem 5. The algebra $A_{4}\left(E_{4}^{\prime}\right)$ is a nil algebra with nil index 4 .

Proof of Theorem 5. For a matrix $A \in A M_{4}\left(E_{4}^{\prime}\right)$, where

$$
A=\left(\begin{array}{cccc}
y_{1} & 0 & z_{1} & 0 \\
0 & y_{2} & 0 & z_{2} \\
z_{3} & 0 & y_{3} & 0 \\
0 & z_{4} & 0 & y_{4}
\end{array}\right)
$$

and $A^{3}=\left(a_{i j}\right)$ we get

$$
\begin{aligned}
& a_{11}=z_{1} z_{3} y_{1}+y_{1} z_{1} z_{3}+z_{1} y_{3} z_{3}, \\
& a_{13}=y_{1}^{2} z_{1}+z_{1} z_{3} z_{1}+y_{1} z_{1} y_{3}+z_{1} y_{3}^{2}, \\
& a_{22}=z_{2} z_{4} y_{2}+y_{2} z_{2} z_{4}+z_{2} y_{4} z_{4}, \\
& a_{24}=y_{2}^{2} z_{2}+z_{2} z_{4} z_{2}+y_{2} z_{2} y_{4}+z_{4} y_{4}^{2}, \\
& a_{31}=z_{3} y_{1}^{2}+y_{3} z_{3} y_{1}+z_{3} z_{1} z_{3}+y_{3}^{2} z_{3}, \\
& a_{33}=z_{3} y_{1} z_{1}+y_{3} z_{3} z_{1}+z_{3} z_{1} y_{3}, \\
& a_{42}=z_{4} y_{2}^{2}+y_{4} z_{4} y_{2}+z_{4} z_{2} z_{4}+y_{4}^{2} z_{4}, \\
& a_{44}=z_{2} y_{2} z_{2}+y_{4} z_{4} z_{1}+z_{4} z_{2} y_{4} .
\end{aligned}
$$

Now we investigate the entries of $A^{4}=\left(b_{i j}\right)$ :

$$
\begin{aligned}
b_{11} & =z_{1} z_{3} y_{1}^{2}+y_{1} z_{1} z_{3} y_{1}+z_{1} y_{3} z_{3} y_{1}+y_{1}^{2} z_{1} z_{3} \\
& +z_{1} z_{3} z_{1} z_{3}+y_{1} z_{1} y_{1} z_{3}+z_{1} y_{3}^{2} z_{3}
\end{aligned}
$$

Applying Corollary 2 we simplify $b_{11}$ and get $b_{11}=z_{1} y_{3} z_{3} y_{1}+y_{1} z_{1} y_{3} z_{3}$. The identity $x y z=-z y x$ gives

$$
z_{1} y_{3} z_{3} y_{1}=-z_{3} y_{1} y_{3} z_{1}=y_{3} z_{1} y_{1} z_{3}=-y_{1} z_{1} y_{3} z_{3} \text {. }
$$

Thus $b_{11}=0$.

In an analogous way we investigate the other entries of $A^{4}$ :

$$
\begin{aligned}
b_{13} & =z_{1} z_{3} y_{1} z_{1}+y_{1} z_{1} z_{3} z_{1}+z_{1} y_{3} z_{3} z_{1}+y_{1}^{2} z_{1} y_{3} \\
& +z_{1} z_{3} z_{1} y_{3}+y_{1} z_{1} y_{3}^{2}+z_{1} y_{3}^{3}
\end{aligned}
$$

According to Corollary 2 we have $b_{13}=0$. 
Now we consider

$$
\begin{aligned}
b_{22} & =z_{2} z_{4} y_{2}^{2}+y_{2} z_{2} z_{4} y_{2}+z_{2} y_{4} z_{4} y_{2}+y_{2}^{2} z_{2} z_{4} \\
& +z_{2} z_{2} z_{2} z_{4}+y_{2} z_{2} y_{4} z_{4}+z_{4} y_{4}^{2} z_{4} .
\end{aligned}
$$

The same Corollary leads to $b_{22}=z_{2} y_{4} z_{4} y_{2}+y_{2} z_{2} y_{4} z_{4}$. As

$$
z_{2} y_{4} z_{4} y_{2}=-z_{4} y_{2} y_{4} z_{2}=y_{4} z_{2} y_{2} z_{4}=-y_{2} z_{2} y_{4} z_{4}
$$

we get $b_{22}=0$.

Applying Corollary 2 we get $b_{24}=b_{31}=0$. In $b_{33}$ we have to consider only the part $y_{3} z_{3} y_{1} z_{1}+z_{3} y_{1} z_{1} y_{3}$. As

$y_{3} z_{3} y_{1} z_{1}=-y_{1} z_{1} z_{3} y_{3}=z_{3} z_{1} y_{1} y_{3}=-y_{3} y_{1} z_{3} z_{1}=z_{1} y_{1} z_{3} y_{3}=-z_{3} y_{1} z_{1} y_{3}$ we get $b_{33}=0$. 0 .

The identities in Corollary 2 immediately lead to $b_{42}=0, b_{44}=0$. Thus $A^{4}=$

Now we consider the subalgebra $\mathrm{ASM}_{4}(E)$ of the matrices of type $\left(\begin{array}{llll}a & 0 & a & 0 \\ 0 & b & 0 & b \\ c & 0 & c & 0 \\ 0 & d & 0 & d\end{array}\right)$ and prove that it is a PI-algebra.

Theorem 6. The algebra $A S M_{4}(E)$ satisfies the identity $U[X, Y, Z]=0$.

Proof of Theorem 6. Let $X, Y, Z$ be matrices from $A S M_{4}(E)$ denoting its entries by $a_{i}, b_{i}, c_{i}, d_{i}$ for $i=1,2,3$ respectively. We form the diagonal entries of $[X, Y]=$ $\left(a_{i j}\right)$, namely

$$
\begin{aligned}
& a_{11}=\left[a_{1}, a_{2}\right]+a_{1} c_{2}-a_{2} c_{1}, \\
& a_{22}=\left[b_{1}, b_{2}\right]+b_{1} d_{2}-b_{2} d_{1}, \\
& a_{33}=\left[c_{1}, c_{2}\right]+c_{1} a_{2}-c_{2} a_{1}, \\
& a_{44}=\left[d_{1}, d_{2}\right]+d_{1} b_{2}-d_{2} b_{1} .
\end{aligned}
$$

For the matrix $[X, Y, Z]=\left(b_{i j}\right)$ we have modulo $[x, y, z]=0$ for $x, y, z \in E$ that

$$
\begin{aligned}
b_{11} & +b_{33} \\
& =\left[a_{1} c_{2}-a_{2} c_{1}, a_{3}\right]+\left(\left[a_{1}, a_{2}\right]+a_{1} c_{2}-a_{2} c_{1}\right) c_{3}-a_{3}\left(\left[c_{1}, c_{2}\right]+c_{1} a_{2}-c_{2} a_{1}\right) \\
& +\left[c_{1} a_{2}-c_{2} a_{1}, c_{3}\right]+\left(\left[c_{1}, c_{2}\right]+c_{1} a_{2}-c_{2} a_{1}\right) a_{3}-c_{3}\left(\left[a_{1}, a_{2}\right]+a_{1} c_{2}-a_{2} c_{1}\right) \\
& =\left[a_{1} c_{2}-a_{2} c_{1}, a_{3}\right]+\left(a_{1} c_{2}-a_{2} c_{1}\right) c_{3}-a_{3}\left(c_{1} a_{2}-c_{2} a_{1}\right) \\
& +\left[c_{1} a_{2}-c_{2} a_{1}, c_{3}\right]+\left(c_{1} a_{2}-c_{2} a_{1}\right) a_{3}-c_{3}\left(a_{1} c_{2}-a_{2} c_{1}\right) \\
& =\left[a_{1} c_{2}-a_{2} c_{1}, a_{3}\right]+\left[c_{1} a_{2}-c_{2} a_{1}, c_{3}\right]+\left[a_{1} c_{2}-a_{2} c_{1}, c_{3}\right]+\left[c_{1} a_{2}-c_{2} a_{1}, a_{3}\right] \\
& =\left[\left[a_{1}, c_{2}\right]+\left[c_{1}, a_{2}\right], a_{3}\right]+\left[\left[c_{1}, a_{2}\right]+\left[a_{1}, c_{2}\right], c_{3}\right] \equiv 0 .
\end{aligned}
$$


Analogously we get that $b_{22}+b_{44}=0$. Thus $U[X, Y, Z]=0$ for any matrix $U \in \mathrm{ASM}_{4}(E)$.

The analogue of $\operatorname{ASM}_{4}(E)$ in the general case is the matrix algebra $A S M_{2 n}(E)$. Its elements are of type $\left(a_{i j}\right)$, where $a_{i i}=a_{i, n+i}$ for $i=1, \ldots, n$ and $a_{j j}=a_{j, j-n}$ for $j=n+1, \ldots, 2 n$. The algebra $A S M_{2 n}(E)$ satisfies the same identity $U[X, Y, Z]=0$.

For now we are able to find involutions in $M_{n}(E)$ for $n>2$ only considering an involution in $E$. We generalize the case $n=2$, namely

Proposition 6. The mapping (b), defined as

$$
\begin{aligned}
& \left(\begin{array}{llll}
a_{11} & a_{12} & a_{13} & a_{14} \\
a_{21} & a_{22} & a_{23} & a_{24} \\
a_{31} & a_{32} & a_{33} & a_{34} \\
a_{41} & a_{42} & a_{43} & a_{44}
\end{array}\right) \\
& =\left(\begin{array}{cc}
A & B \\
C & D
\end{array}\right)^{(b)}=\left(\begin{array}{cc}
(D)^{b} & (B)^{b} \\
(C)^{b} & (A)^{b}
\end{array}\right)=\left(\begin{array}{llll}
a_{44}^{*} & a_{34}^{*} & a_{24}^{*} & a_{14}^{*} \\
a_{43}^{*} & a_{33}^{*} & a_{23}^{*} & a_{13}^{*} \\
a_{42}^{*} & a_{32}^{*} & a_{22}^{*} & a_{12}^{*} \\
a_{41}^{*} & a_{31}^{*} & a_{21}^{*} & a_{11}^{*}
\end{array}\right)
\end{aligned}
$$

is an involution on $M_{4}(E, \psi=*)$.

Proof of Proposition 6. Considering in details the entries of the two matrices $(A B)^{(b)}$ and $(B)^{(b)}(A)^{(b)}$ we see that their corresponding entries are equal i.e. the mapping (b) is an involution.

We cover the following special case: Let $E_{3}^{\prime}$ be the non-unitary finite dimensional Grassmann algebra with generators $e_{1}, e_{2}, e_{3}$ and $A M(2)\left(E_{3}^{\prime}\right)$ be the subalgebra of $A M_{4}\left(E_{3}^{\prime}\right)$ defined by the matrices of type

$$
\left(\begin{array}{cccc}
y_{1} & 0 & z_{1} & 0 \\
0 & y_{2} & 0 & z_{2} \\
z_{3} & 0 & y_{3} & 0 \\
0 & z_{4} & 0 & y_{4}
\end{array}\right),
$$

where $y_{i}$ are even elements (of even length) of $E_{3}^{\prime}$, while $z_{i}$ are odd elements (of odd length) of $E_{3}^{\prime}, i=1, \ldots, 4$. We equip the algebra $A M(2)\left(E_{3}^{\prime}, \psi_{2}\right)$ with the involution (b) as defined in Proposition 6.

We characterize the (b)-symmetric elements $Y_{i}$ and the (b)-skew symmetric elements $Z_{j}$ of the algebra $\left(A M(2)\left(E_{3}^{\prime}, \psi_{2}\right),(b)\right)$.

Theorem 7. The algebra $\left(A M(2)\left(E_{3}^{\prime}, \psi_{2}\right),(b)\right)$ satisfies the (b)-identity $Y^{3}=0$ in (b)-symmetric variables. 
Proof of Theorem 7. Let consider a (b)-symmetric element $Y$. Denoting for short $\psi_{2}$ as $*$ in the equality $\left(\begin{array}{cccc}y_{4}^{*} & 0 & z_{2}^{*} & 0 \\ 0 & y_{3} * & 0 & z_{1}^{*} \\ z_{4} * & 0 & y_{2}^{*} & 0 \\ 0 & z_{3} * & 0 & y_{1}^{*}\end{array}\right)=\left(\begin{array}{cccc}y_{1} & 0 & z_{1} & 0 \\ 0 & y_{2} & 0 & z_{2} \\ z_{3} & 0 & y_{3} & 0 \\ 0 & z_{4} & 0 & y_{4}\end{array}\right)$ we get the following conditions on the entries of $Y: \psi_{2}\left(y_{4}\right)=y_{1}, \psi_{2}\left(y_{3}\right)=y_{2}, \psi_{2}\left(z_{2}\right)=$ $z_{1}$ and $\psi_{2}\left(z_{4}\right)=z_{3}$.

Let $y_{1}=s_{1} e_{1} e_{2}+s_{2} e_{1} e_{3}+s_{3} e_{2} e_{3}$. Then $y_{4}=\psi_{2}\left(y_{1}\right)=-y_{1}$. For $y_{2}=t_{1} e_{1} e_{2}+$ $t_{2} e_{1} e_{3}+t_{3} e_{2} e_{3}$ we get $y_{3}=\psi_{2}\left(y_{2}\right)=-y_{2}$. Obviously $y_{1}^{2}=y_{2}^{2}=0$.

As the entries are from $E_{3}^{\prime}$ we could work with odd entries having summands of degree 1 only. Let $z_{1}=\alpha_{1} e_{1}+\alpha_{2} e_{2}+\alpha_{3} e_{3}$ and $z_{3}=m_{1} e_{1}+m_{2} e_{2}+m_{3} e_{3}$. Then $z_{2}=\psi_{2}\left(z_{1}\right)=z_{1}, z_{4}=\psi_{2}\left(z_{3}\right)=z_{3}$. Considering $Y^{3}=Y^{2} Y=\left(a_{i j}\right)$ as

$$
\left.\begin{array}{cccc}
z_{1} z_{3} & 0 & y_{1} z_{1}-z_{1} y_{2} & 0 \\
0 & z_{1} z_{3} & 0 & y_{2} z_{1}-z_{1} y_{1} \\
z_{3} y_{1}-y_{2} z_{3} & 0 & z_{3} z_{1} & 0 \\
0 & z_{3} y_{2}-y_{1} z_{3} & 0 & z_{3} z_{1}
\end{array}\right)
$$

we see that manipulating with the generators $e_{1}, e_{2}, e_{3}$, probably nontrivial entries could be only

$$
a_{13}=a_{24}=z_{1} z_{3} z_{1}=\beta_{1} e_{1} e_{2} e_{3}, a_{31}=a_{42}=z_{3} z_{1} z_{3}=\beta_{2} e_{1} e_{2} e_{3} .
$$

Applying Corollary 2 we get that both of them are zero.

Theorem 8. The algebra $\left(A M(2)\left(E_{3}^{\prime}, \psi_{2}\right),(b)\right)$ satisfies the (b)-identity $Z^{3}=0$ in (b)-skew symmetric variables.

Proof of Theorem 8. Using the same notations for the matrix entries of $Z$ as in the previous theorem, in this case we have

$$
\begin{aligned}
& y_{4}=-\psi_{2}\left(y_{1}\right)=y_{1}, y_{3}=-\psi_{2}\left(y_{2}\right)=y_{2}, \\
& y_{1}^{2}=y_{2}^{2}=0, \\
& z_{2}=-\psi_{2}\left(z_{1}\right)=-z_{1}, z_{4}=-\psi_{2}\left(z_{3}\right)=-z_{3} .
\end{aligned}
$$

In $Z^{3}=Z^{2} Z=\left(b_{i j}\right)$ nonzero could be only the entries $b_{13}=-b_{24}=z_{1} z_{3} z_{1}$ and $b_{31}=-b_{42}=z_{3} z_{1} z_{3}$. Corollary 2 proves they both are zero.

We consider the subalgebra $\left(A M(2)\left(E_{3}^{\prime}, \psi_{2}\right),(b)\right)$ instead of the algebra $\left(A M_{4}\left(E_{3}^{\prime}, \psi_{2}\right),(b)\right)$ itself as if $A^{n}=0$ for a b-variable $A$ of $\left(A M_{4}\left(E_{4}^{\prime}, \psi_{2}\right),(b)\right)$ we 
have $n>3$. Thus the algebras $\left(A M_{4}\left(E_{4}^{\prime}, \psi_{2}\right),(b)\right)$ and $A M_{4}\left(E_{4}^{\prime}\right)$ have equal nil indices.

We give an example of another matrix algebra with involution (b) having lower nilpotency index of its (b)-skew symmetric variables:

Let $B M(2)\left(E_{3}^{\prime}\right)$ be the algebra defined by the matrices of type

$$
\left(\begin{array}{cccc}
y_{1} & 0 & 0 & z_{1} \\
0 & y_{2} & z_{2} & 0 \\
0 & z_{3} & y_{3} & 0 \\
z_{4} & 0 & 0 & y_{4}
\end{array}\right)
$$

, where $y_{i}$ are even elements of $E_{3}^{\prime}$, while $z_{i}$ are odd elements of $E_{3}^{\prime}, i=1, \ldots, 4$. We equip the algebra $B M(2)\left(E_{3}^{\prime}, \psi_{2}\right)$ with the involution (b) as defined in Proposition 6.

Theorem 9. The algebra $\left(B M(2)\left(E_{3}^{\prime}, \psi_{2}\right),(b)\right)$ satisfies the $(b)$-identity $Y^{3}=0$ in (b)-symmetric variables and the (b)-identity $Z^{2}=0$ in (b)-skew symmetric variables.

Proof of Theorem 9. In the algebra $\left(B M(2)\left(E_{3}^{\prime}, \psi_{2}\right),(b)\right)$ any (b)-skew symmetric variable $Z$ is a diagonal matrix and $Z^{2}=0$ as $y_{i}^{2}=0$ for $i=1, \ldots, 4$.

There is a package written in the system for computer algebra Mathematica [10] for manipulating in finite dimensional Grassmann algebras. Using it a programme was written by the author giving an alternative way of confirming the validity of the corresponding theorems in the paper.

\section{REFERENCES}

[1] S. Amitsur, "Identities in rings with involution," Izrael J. of Mathematics, vol. 7, pp. 63-68, 1969, doi: 10.1007/BF02771748.

[2] N. Anisimov, "Codimensions of identities with the Grassmann algebra involution," Mosc. Univ. Math. Bull., vol. 56, no. 3, pp. 25-29, 2001.

[3] A. Berele and A. Regev, "Exponential growth for codimensions of some P.I. algebras," J. Algebra, vol. 241, pp. 118-145, 2001, doi: 10.1006/jabr.2000.8672.

[4] O. Di Vincenzo, "On the graded identities of $M_{1,1}(E)$, , Israel J. Math., vol. 80, pp. 323-335, 1992, doi: 10.1007/BF02808074.

[5] V. Drensky and A. Giambruno, "On the *-polynomial identities of minimal degree for matrices with involution," Boll. Unione Math. Ital. A(7), vol. 9, no. 3, pp. 471-482, 1995.

[6] A. Giambruno and A. Valenti, "On minimal *-identities of matrices," Linear and Multilin. Algebra, vol. 39, pp. 309-323, 1995, doi: 10.1080/03081089508818405.

[7] D. Krakowski and A. Regev, "The polynomial identities of the Grassmann algebra," Trans. Amer. Math. Soc., vol. 181, pp. 429-438, 1973, doi: 10.2307/1996643.

[8] D. La Mattina and P. Misso, "Algebras with involution and linear codimendion growth," $J . A l$ gebra, vol. 305, pp. 270-291, 2006, doi: 10.1016/j.jalgebra.2006.06.044.

[9] D. Levchenko, "Finite basis of identities with an involution for the second order matrix algebra (in Russian)," Serdica Math. J., vol. 8, no. 1, pp. 42-56, 1982.

[10] A. Mihova and T. Rashkova, "Usage of Mathematica in manipulating with Grassmann entries (in Bulgarian)," Proc. of Ruse University, ser. 5.1, vol. 41, pp. 22-27, 2008. 
[11] T. Rashkova, "Involution matrix algebras - identities and growth," Serdica Math. J., vol. 30, no. 2-3, pp. 239-282, 2004.

[12] T. Rashkova, "Nilpotency in involution matrix algebras over algebra with involution," Mathematics and Education in Mathematics, pp. 143-150, 2009.

[13] T. Rashkova, "Matrix algebras over Grassmann algebras and their PI-structure," Acta Universitatis Apulensis, Special Issue, pp. 169-184, 2011.

[14] L. Rowen, "A simple proof of Kostant's theorem and an analogue for the symplectic involution," Contemp. Math., vol. 13, pp. 207-215, 1982.

[15] S. Tumurbat and R. Wiegandt, "A-radicals of involution rings," South Asian Bull. Math., vol. 29, no. 2, pp. 393-399, 2005.

[16] U. Vishne, "Polynomial identities of $M_{n}(G)$," Communs. in Algebra, vol. 30, no. 1, pp. 443-454, 2002.

[17] M. Wenxin and M. Racine, "Minimal identities of symmetric matrices," Trans. Amer. Math. Soc., vol. 320, no. 1, pp. 171-192, 1990, doi: 10.1090/S0002-9947-1990-0961598-6.

\section{Author's address}

\section{Tsetska Rashkova}

University of Ruse, Department of Mathematics, 8 Studentska Str., 7017 Ruse, Bulgaria

E-mail address: tsrashkova@uni-ruse.bg 